\title{
The relativity of mind from physical viewpoint
}

\author{
K. Wang, *
}

\begin{abstract}
The mind and consciousness are pretty complex to observe and analyze quantitatively since the complexity of neuron and mystery of consciousness. Recent years, many scientists try to explain our mind from physical viewpoint or elucidate the physics of the mind. In the paper, we reveal a new approach to understand our mind and present a tentative theory to describe how our mind works respect to physics. Two significant concept of time, namely, the internal time of mind and external time of the outside are introduced to measure our mind quantitatively on some degree. The related law of these two types of time is similar to the special relativity proposed by Einstein. Our theory aims to provide a new perspective to understand and know ourselves and lead our life better. At the end of paper, we design an experiment to check the correctness of our results.
\end{abstract}

Introduction. One of the most difficult things for us is to know ourselves, especially our mind. As we all know, although the biotechnology has developed rapidly, the mind is so hard to detect using machine, and the most useful way to observe our mind is through our feelings unitil now. On the other hand, we have known that the consciousness comes from the complex relation between billions of neurons, but it is not clear how they work indeed. Some bioscientists are trying to detect our brain waves to observe how our mind works [1-3], however, it seems no much promotion. The reason is obvious, human is too complex, it is not enough to merely study neurons to understand consciousness, though which arises from neurons, let alone find a universal theory to explain our mind completely. Some other scientists begin to look for the physics of the mind and try to explain the mind from physical viewpoint, for instance, quantum mind and entangled consciousness [4-13]. Besides, the relation between the mind and the behaviors is still a mysetry. Though the behaviors are decided by mind, it is unclear how mind determines behaviors. Freud tried to understand our behaviors by studying our dreams in his famous work The Interpretation of Dreams [14].

In this paper, we attempt to build a new path to understand our mind and behaviors from a novel physical viewpoint. We define two kinds of time, namely, the Internal Time and Space (ITS), which is the abstract spacetime in our mind depending on our consciousness since we even can not observe it but feel it, and the External Time and Space (ETS), which is just the spacetime where we live in. With these definitions, we can elucidate our main result of this paper, namely, The Relativity of Mind. Then we proceed to discuss a relation between mind and behaviors with respect to time, consisting of the Ideological Behaviors Theory, the Inertia of the Behaviors with Mind and Its Corollary.

The relativity of mind. With respect to ITS and ETS, we elaborate the following empirical law. When we are thinking, the faster our mind thinks, the slower the time of ITS lapses, and comparatively, the faster the time of ETS, and vice versa. As aforementioned, the ITS is the abstract spacetime in our mind, which we can not touch or observe, but feel it with our active consciousness. The consciousness is exactly the difference between human and animals, in fact, it is the privilege of people. We seperate the ITS and ETS because they are in different spacetime. For convenient analysis and intuitive observation, inspired by the special relativity of Einstein [15], we give the following not very accurate and unproved formula to elaborate our thoughts qualitatively,

$$
\frac{\Delta t_{I}}{\Delta t_{E}}=\Phi(V)=\frac{V_{0}}{V} \sqrt{\frac{C^{2}-V^{2}}{C^{2}-V_{0}^{2}}},
$$

where $V$ is the thinking speed of ITS, it is actually a function of human's states, especially human's brain, even related to every neurons. $\Delta t_{I}$ is the time of ITS, $\Delta t_{E}$ is the time of ETS. $C$ is the maximum of $V$, which means that the human's thinking speed has an extreme value, for everyone. But different people may have different maximum $C$. Maybe when your total billions of neurons are working at the fastest speed, your thinking speed can reach the maximum. We plot the function in Fig. 1. Nevertheless, people can't reach this level with the current level of human's intelligence, even can not reach it forever, it is just an ideal maximum value. Like the speed of light in the universe, unless the mass of object is 0 , its speed of motion will never reach the speed of light. According to the description of the theory and the qualitative equation (1), we can derive the following resluts: When $V$ is equal to 0 , the rate $\frac{\Delta t_{I}}{\Delta t_{E}}$ is infinity, because in this case, people can be taken as dead; When $V$ is equal to the value $V_{0}$, the rate $\frac{\Delta t_{I}}{\Delta t_{E}}$ is 1 . In this case, the speed of human's internal and external time is equal; When $V$ reaches the maximum $C$, the rate $\frac{\Delta t_{I}}{\Delta t_{E}}$ is 0. In this case, it is no exaggeration to say that the person is eternal.

Another important question is how to measure the time and the thinking speed of ITS. This is a very difficult problem. Because this kind of measurement can't be done by outside people, only ourselves can do. But our own measurements are not actually accurate and objective. Thus we say, the theory depends on the development of science and technology. We hope more people will do reseach in this area in the future and develop the theory more accurate and useful. So far, we take the idea 


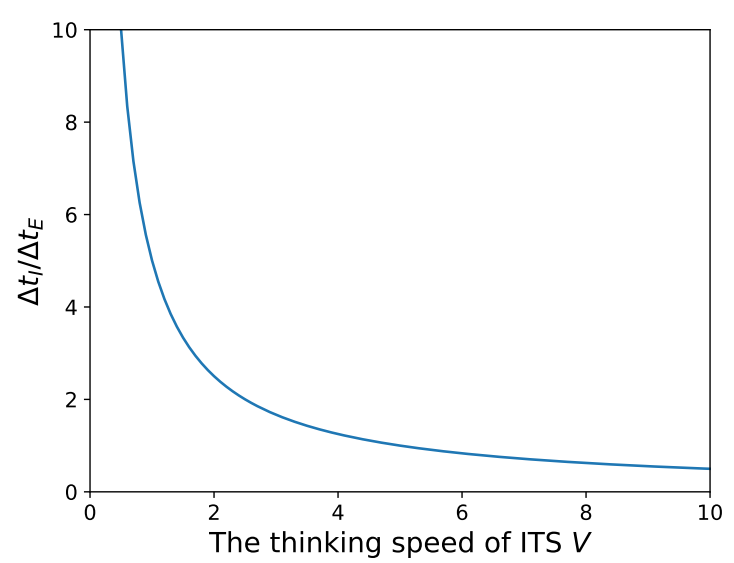

FIG. 1: A qualitative description for the relativity of mind. The parameters are taken as $V_{0}=5, C=100$.

that the amount of information that appears in our brain per unit time can be taken as the thinking speed of ITS, which may be measured by detecting the brain waves of human in experiments. Nevertheless, the time of ITS is just can be felt by people up to now.

We now take some examples in daily life to elaborate the result. If you are burying yourself in a math text or reading a novel seriously, or thinking about a scene quickly, when the outside world has passed two hours, you may only feel just one hour, since your thinking speed of ITS is faster than $V_{0}$; If there are ten minutes left in class, but you have nothing to do, only one thought in your mind at that time, "Hurry up, let's get out of class!" and you often look your watch, then after class, maybe you think it is half an hour because your thinking speed of ITS is slower than $V_{0}$. By the way, for the cases of actively accepting outside information, it is also suitable. For example, when watching TV, the brain constantly inputs images and texts from the screen, and they enter the brain to form "brain memory", which is equivalent to increasing their thinking speed, and on the contrast, if you are looking at videos that are not of interest, then those information can not input your brain and can not increase your thinking speed indeed. Interestingly, in this way, the judgement of people's lifespan can not merely depends on the superficial lifespan, because there is an internal time in everyone mind, which is the more realistic lifespan in fact.

The ideological behaviors theory. As for the relation between mind and behaviors, we have the following conclusion. In unit time, for instance one minute, a person is in a certain state, which means the energy consumed by this person at that time is certain, then the speed of motion of this person's behaviors (SMB) is inversely related to the thinking speed of ITS. In other words, the higher the SMB (which means the more you move in a certain period of time), the lower the thinking speed of ITS. Conversely, the lower the SMB, the higher the thinking speed

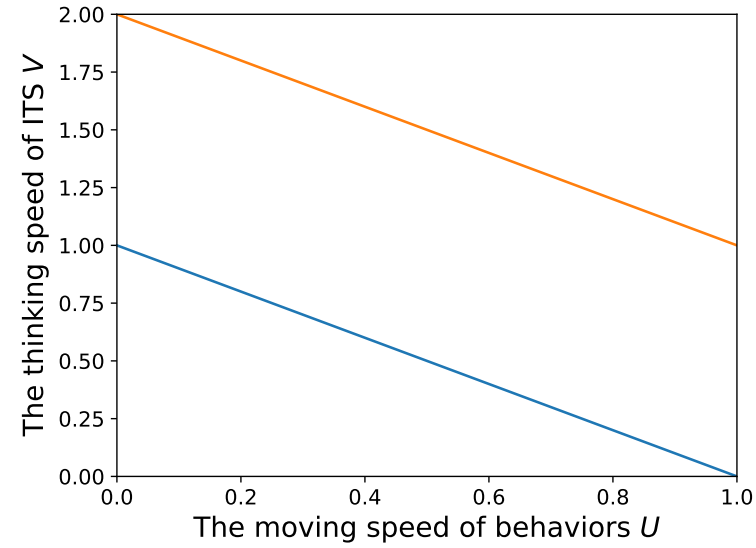

FIG. 2: A qualitative description for the relation of mind and behaviors. The parameters are taken as $V_{\max }=1, U_{\max }=1$ for the below blue line and $V_{\max }=2, U_{\max }=2$ for the upper orange line. If the below line represents the common state of a person, then the upper line represents the lift of the common state. For example, the person secretes more adrenaline.

of ITS. This relation can be described by the following qualitative formula,

$$
\frac{V}{V_{\max }}+\frac{U}{U_{\max }}=1
$$

where $V$ is the thinking speed of ITS and $U$ moving speed of behaviors. Note that for different level of human's states, $V_{\max }$ and $U_{\max }$ can have differnet values. Namely, when human's state is lifted, for example, the person secretes more adrenaline, then the values of $V_{\max }$ and $U_{\max }$ will get bigger. The corresponding figure for explicit explanation is shown in Fig. (2).

For example, when you are doing violent exercise, your SMB will become larger, and your thinking speed of ITS will become smaller (assuming that you do not deliberately improve your level of state to increase the thinking speed of ITS), and then according to the relativity of mind, when you feel the time has passed an hour, the outside time may has only lapsed half an hour. Thus we can say in some ways, speaking less and doing more, you can think farther and deeper.

The inertia of the behaviors with thoughts and its corollary. People know that the matter obeys the law of inertia because of its mass, we here reveal that the mind and behaviors have inertia as well: When a person suddenly remembers something else (not related to what he is doing, neither take the initiative to change the current behaviors), and thinks about it with a high degree of concentration, then his body will remain in its original state, i.e., staying still or continue the current behaviors unless there are external conditions to change this state of mind, or outside conditions to block his behaviors, or his mind returns to himself (i.e., wake up from contemplation). The higher the speed of thinking other things, the more stable the original state is, meanwhile 
the higher the conditions required to change the original state. This rule can be qualitatively described by the following simple formula,

$$
\Psi(V)=k V,
$$

where $V$ is the speed of thinking other things, and $\Psi(V)$ represents the degree of condition required to change the state of mind (or change its state of behaviors) under the corresponding $V$. The degree of $\Psi(V)$ is a relatively abstract concept, perhaps the intensity or frequency of the sound, or the strength of the outside world to touch you, etc. In short, it is the minimum degree of external conditions to change the original state. On the other hand, changing the state of mind and physical behaviors are same in some ways, because once the state of mind changes (such as the outside world pulls your meditation back into reality), it can often directly lead to changes in your body's state of behaviors. The change of the state of physical behaviors can often stimulate the change of state of mind, but usually, the former change is relatively easier. For example. When you are looking at a place (like watching a distant landscape), suddenly you think of a mathematical or physical problem, and then desperately want to work out it, then you will maintain this state of the body, the eyes will not even blink, the body will not move. If your thinking speed is relatively slow, even if the sound of an embroidery needle falling to the ground can "awake" you; but if your thinking speed is relatively fast, even someone call you behind, you will not notice that.

The corollary. Based on the above result, we can easily reveal a corollary as below. Under the same external conditions, the faster the thinking speed, the smaller the influence of external conditions on it, the slower the thinking speed, and the greater the influence of external conditions on it. For instance, when a person is doing something, if he is fully engaged, namely his thinking speed is high, the external environment will hardly affect him, even if you call his name or pat him, he may not notice.

The procedure of behaviors and mind. Furthermore, we discuss another situation for behaviors and mind, which is similar to former the inertia of behaviors and mind, but with some difference. If a person is doing something, meanwhile he has been already familiar with the process of working it, then even if he shifts most of his attention away, leaving little attention on it, his that behavior will still work, since an behavior program had been set up in his mind, usually in subconscious, and his behaviors will obey the order of mind. The difference between this one and the above one is the requirement of thinking speed. The previous one usually needs a higher thinking speed and does not need to keep in mind the process of behaviors, and this one does not require a high thinking speed od mind. Another notable point is that the previous one usually maintains relatively simple behaviors such as simple swinging legs, walking or standing still, and the attention at that time is completely removed, on the contrast, the behaviors allowed by this law can be a bit more complicated than the former. However, we say, if the behaviors are too complicated, it will easier go wrong.

Regarding the biological cause of this phenomenon, we have a guess: when an (or a series of) behavior is repeated many times, its behavioral process will form a clear "loop" in the brain and the neurons are connected more closer by synapass. No matter one presses the "switch" from any position in this "loop", the behavior will successfully complete the rest, even without requiring you to actively intervene. In other words, this is like a gradual transformation from conditional reflex to unconditioned reflex. At first, the behavioral process is familiarized by learning behavior, and then you no longer need to intervene. The behavior is completed under a series of subconscious commands. The "loop" in the brain is equivalent to the behavioral procedure. For example, if a man is carrying a box (not too heavy and easy to move), holds it from the top of the car, then takes a distance, and puts it in a designated position. Repeating this work, when after he does it about a dozen times, his mind about the behavioral procedure in the subconscious mind (in fact, the process of writing this program was not human's intervention) will be built, thus even if he is absent-thoughtsed, namely, he takes most of his attention to other things, only very little attention is paid to this behavior, he can still do the job well, and finally he will feel "what hasn't happened yet." This is actually because when he carries most of the boxes, only the behavioral program is working.

Experimental methods. We now design an experiment to verify our theory. Since the theory is applicable to human, and the time of ITS needs our own feelings. We can randomly select 500 or more honest people to do experiments. Let them read some text to calculate the speed of thinking per unit time, (or meanwhile detect their power of brain waves) and then over a period of time, let them say how long they feel. Then we take this time to compare with the actual time, and repeat the experiment, finally we collect statistics of average. This experiment is used to verify the relativity of mind, and the remaining rules can also be verified utilizing the similar method.

Discussion and summary. Here, we discuss a special condition: When people are sleepy, situation maybe a little different. A key point in our theory is that people can feel the time flow, and have an positive consciousness. However, when people is sleepy, he can not feel the time flow actively and has a negative consciousness. At that moment, the subconscious is working mostly. In summary, we reveal the relation between internal spacetime of mind and external spacetime, and connect the mind with behaviors in some ways. We try to link non- 
quantitative life and quantitative natural sciences, and we aim to help people know themselves better.

*wk813943178@gmail.com

[1] B. J. Baars and N. M. Gage, Cognition, Brain, and Consciousness: Introduction to Cognitive Neuroscience (Academic Press; 2nd edition, 2010).

[2] B. Libet, Mind Time: The Temporal Factor in Consciousness (Perspectives in Cognitive Neuroscience) (Harvard University Press, 2005).

[3] S. Menon, Brain, Self and Consciousness (Springer India, 2014).

[4] L. I. Perlovsky, Physics of Life Reviews 3, 23 (2006), URL https://doi.org/10.1016\%2Fj.plrev. 2005.11.003.

[5] S. JM and H. P. Stapp, Preprint pp. 1-72 (2004).

[6] P. Di Sia, Mindfulness, Consciousness and Quantum Physics (2018), URL https://doi.org/10.31219/osf. io/ftx9m.

[7] C. W. Lynn and D. S. Bassett, Nature Reviews Physics 1 (2019), ISSN 2522-5820, URL http://dx.doi.org/10. 1038/s42254-019-0040-8

[8] B. D. Josephson, Activitas Nervosa Superior 61, 86 (2019), ISSN 18029698.

[9] S. Hameroff, in Pensamiento (2011), vol. 67, pp. 641-659, ISSN 00314749.

[10] L. I. Perlovsky, Toward physics of the mind: Concepts, emotions, consciousness, and symbols (2006).

[11] W. Loewenstein, Physics in Mind: A Quantum View of the Brain (Basic Books; 1 edition (January 29, 2013), 2013).

[12] H. Atmanspacher, Quantum Approaches to Consciousness (Metaphysics Research Lab, Stanford University, 2020), summer 2020 ed.

[13] D. Radin, Entangled Minds: Extrasensory Experiences in a Quantum Reality (Paraview Pocket Books; 45834th edition, 2006).

[14] S. Freud, The Interpretation of Dreams, Third Edition. Trans. by A. A. Brill. (New York: The Macmillan Company, 1913., 2010).

[15] A. Einstein, RELATIVITY THE SPECIAL AND GENERAL THEORY (NEW YORK HENRY HOLT AND COMPANY, 1920). 